\title{
Limitations to emission spot size in laser lighting
}

Jensen, Ole Bjarlin; Krasnoshchoka, Anastasiia; Hansen, Anders Kragh; Thorseth, Anders; Marti, Dominik; Jian, Xu; Petersen, Paul Michael

\section{Published in:}

Proceedings of SPIE

Link to article, DOI:

$10.1117 / 12.2542674$

Publication date:

2020

Document Version

Peer reviewed version

Link back to DTU Orbit

Citation (APA):

Jensen, O. B., Krasnoshchoka, A., Hansen, A. K., Thorseth, A., Marti, D., Jian, X., \& Petersen, P. M. (2020). Limitations to emission spot size in laser lighting. In Proceedings of SPIE (Vol. 11302). [113020B] SPIE International Society for Optical Engineering. Proceedings of SPIE - The International Society for Optical Engineering https://doi.org/10.1117/12.2542674

\section{General rights}

Copyright and moral rights for the publications made accessible in the public portal are retained by the authors and/or other copyright owners and it is a condition of accessing publications that users recognise and abide by the legal requirements associated with these rights.

- Users may download and print one copy of any publication from the public portal for the purpose of private study or research.

- You may not further distribute the material or use it for any profit-making activity or commercial gain

- You may freely distribute the URL identifying the publication in the public portal 


\title{
Limitations to emission spot size in laser lighting
}

\author{
Ole Bjarlin Jensen*a , Anastasiia Krasnoshchoka ${ }^{\mathrm{a}}$, Anders Kragh Hansen ${ }^{\mathrm{a}}$, Anders Thorseth ${ }^{\mathrm{a}}$, \\ Dominik Marti ${ }^{\mathrm{b}}, \mathrm{Xu} \mathrm{Jian}^{\mathrm{c}}$, and Paul Michael Petersen ${ }^{\mathrm{a}}$ \\ aDepartment of Photonics Engineering, Technical University of Denmark, Frederiksborgvej 399, \\ 4000 Roskilde, Denmark; 'Department of Health Technology, Technical University of Denmark, \\ Frederiksborgvej 399, 4000 Roskilde, Denmark; ' ${ }^{\circ}$ ab of New Energy Materials and Devices, School \\ of Physics and Electronic Information, Henan Polytechnic University, Jiaozuo 454000, China
}

\begin{abstract}
Laser lighting is an emerging technology to generate high luminance lighting. To achieve high luminance or high luminous exitance, the light emitter must have high flux and small size simultaneously. When laser light is focused to a small spot size on the phosphor material, the two main limitations are saturation of the phosphor material and the spot size of the generated light. Here, we investigate experimentally and numerically the spot size of laser lighting dependent on the spot size of the incident laser light and the material properties of the wavelength converting phosphor material. We find numerically that the spot size of the generated white light is significantly influenced by the phosphor properties. The spot size of the white light determines the étendue and thereby the possibility to collect and shape the light. This has important implications in applications of laser lighting.
\end{abstract}

Keywords: Laser lighting, spot size, phosphor materials,

\section{INTRODUCTION}

In laser lighting, a blue laser is illuminating a phosphor material to convert some of the incident blue light to longer wavelengths and thereby achieve white light by mixing with the remaining blue light. Lasers operate by stimulated emission and, therefore, do not suffer from the efficiency droop experienced in LEDs [1]. This means that significantly higher power density can be extracted from laser diodes than from LEDs. Focusing of the blue laser light onto a wavelength converting material, typically a phosphor, will generate high intensity white light. Application of laser lighting is rapidly growing due to the possibility to achieve high luminous flux from a small area. This high luminous exitance enables generation of well-collimated white light, which finds application in various fields such as endoscopy [2], car head lights [3] and projection [4].

Focusing of high power blue laser light onto a phosphor material has certain limitations. One limitation is the durability of the phosphor materials. The phosphor materials used for LEDs are typically based on organic resins. These materials are not well suited for laser illumination due to low thermal conductivity and these phosphors typically carbonize when used with lasers. More durable materials like single crystals, ceramic material and phosphor in glass show great promise as materials capable of handling high laser power [5-9]. One of the main limitations is saturation of the luminescence from the phosphor. The energy of the generated photons is lower than for the incident photons. This energy difference is termed the Stokes loss and this loss will generate heat. A phosphor material is not converting all the absorbed blue light to longer wavelengths. The quantum efficiency of a phosphor material describes the efficiency in converting absorbed photons to luminescent photons. The excess absorbed photons will generate heat [10]. The quantum efficiency is temperature dependent with lower efficiency at elevated temperature $[11,12]$. When the phosphor material is heated by the incident blue laser light, the efficiency drops. This drop is termed thermal quenching and has been investigated in several phosphor materials as limiting the luminous flux under high intensity laser illumination [6,13-16].

Another limitation to high intensity white light, which has not been extensively studied, is the spot size of the generated luminescent light. The phosphor material absorbs blue light in a certain volume, from which luminescence is emitted. The size of this volume depends on the surface properties and the scattering and absorption properties of the material.

*ojen@ fotonik.dtu.dk; phone +45 46774553; Fotonik.dtu.dk 
The luminescent light is emitted isotropically in all directions and the luminescent spot will be larger than the spot of the incident blue light. The main focus of most other reported investigations has been on modeling the thermal distribution in the phosphor to limit saturation $[2,17,18]$. One attempt to limit thermal quenching was reported by Ma et al. with simulation of the temperature distribution and experimental verification [19]. In a recent reporting, Zhang et al. investigated the impact of scattering on the luminescent spot [20]. Using different pore structures in ceramic phosphors, they investigated the influence on the luminescent spot.

Here, we numerically and experimentally investigate the relationship between the incident blue laser spot size and the luminescent spot size in different phosphor materials. Experimentally, we investigate the luminescent spot size in a ceramic phosphor material. We use a Monte Carlo model to simulate the light distribution in phosphor materials with different scattering and absorption properties. We find that the luminescent spot size depends strongly on the material properties, the surface structure as well as the thickness and configuration, in which the phosphor is used. The experimental and simulated results are in good agreement.

\section{MONTE CARLO MODEL}

A blue photon incident on a phosphor material can experience different scenarios during propagation. It can be reflected or back-scattered at the surface; it can enter the phosphor and be transmitted or scattered without absorption; it can be absorbed with or without previous scattering. The luminescent light is emitted from the point, where a blue photon is absorbed. The luminescent light is emitted isotropically and may then undergo scattering and absorption in the material and be reflected or transmitted at the edges of the material. The phosphors were modelled as consisting of two layers. The first layer is the phosphor material and the second layer is either air in transparent phosphors or a mirror layer in reflective phosphors.

We used the Monte Carlo method including fluorescence to model the light propagation in the phosphor materials. Monte Carlo methods are accurate and flexible for modeling light transport in scatting and absorbing media. In our work, the model is symmetric. We chose to use the new software suite MCmatlab, which is capable of modeling light distribution and includes fluorescence [21].

For the modeling, we assumed the scattering coefficients $\left(\mu_{\mathrm{a}}\right)$ and the scattering anisotropy $(\mathrm{g})$ for the incident blue light and the luminescent light to be identical and that the absorption coefficient $\left(\mu_{\mathrm{a}}\right)$ of the luminescent light is negligible. Furthermore, we assumed the luminescent light to consist of only one wavelength. Here we used $580 \mathrm{~nm}$ as a representative value for the luminescent light. Previously, it was shown that these assumptions are valid [22]. The parameters for scattering and absorption for the different phosphor materials were extracted from the literature [13,23] and to mimic the properties of different phosphor materials. The values used for the modeling are given in Table 1 . Air was modeled as completely transparent, while a mirror was assumed to reflect all photons following the law of reflection.

The incident blue light was assumed to have a Gaussian beam profile on the surface of the phosphor. The surface properties of the phosphor determined the beam profile of the blue light, when it enters the phosphor. For a plane phosphor surface, the input Gaussian beam was allowed to propagate unchanged through the surface, while for a highly scattering surface, we assumed a Lambertian distribution of the blue light inside the phosphor.

In order to reach a smooth photon distribution at the observation plane, a sufficient number of photons must be launched. For each size of the input blue laser spot, the simulation volume was chosen to ensure only few photons would exit the simulation volume at the side boundaries.

In the first part of the model, we model the distribution and absorption of the blue light. An example of the blue light distribution inside a $250 \mu \mathrm{m}$ thick scattering phosphor with rough surface and $\mathrm{g}=0.75, \mu_{\mathrm{a}}=40 \mathrm{~cm}^{-1}$ and $\mu_{\mathrm{s}}=100 \mathrm{~cm}^{-1}$ is given in Figure 1. When the blue light distribution is known, the position of the luminescent emitters is also known and the second part of the model will perform a Monte Carlo simulation of the luminescent light distribution. The luminescent light is generated isotropically in all directions and an example of the luminescent light distribution inside the phosphor is also shown in Figure 1. As seen, the luminescent light distribution is significantly wider than the input blue light distribution and filling out the phosphor in the vertical direction. In this work we were mainly interested in the distribution of the luminescent light going out of the input surface of the phosphor materials. This distribution is shown in Figure 2. Again, it is seen that the luminescent light distribution is wider than the input blue light distribution. 

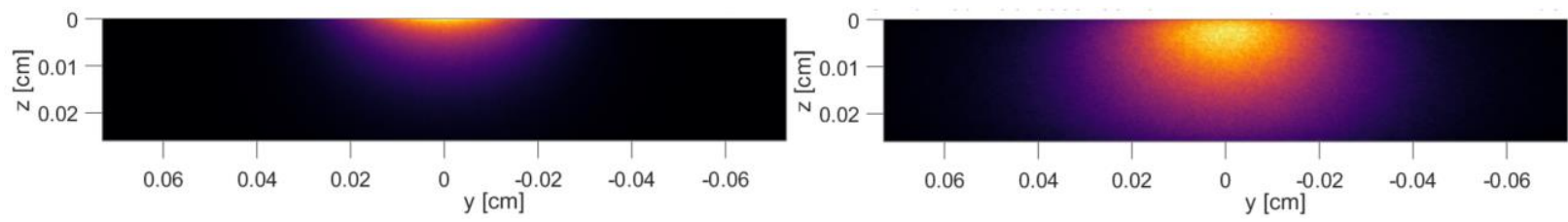

Figure 1. Normalized fluence rate of the blue light inside a phosphor material with the blue light entering from the top (left). Normalized luminescent light fluence rate inside a phosphor material (right). See Figure 2 for color scale.
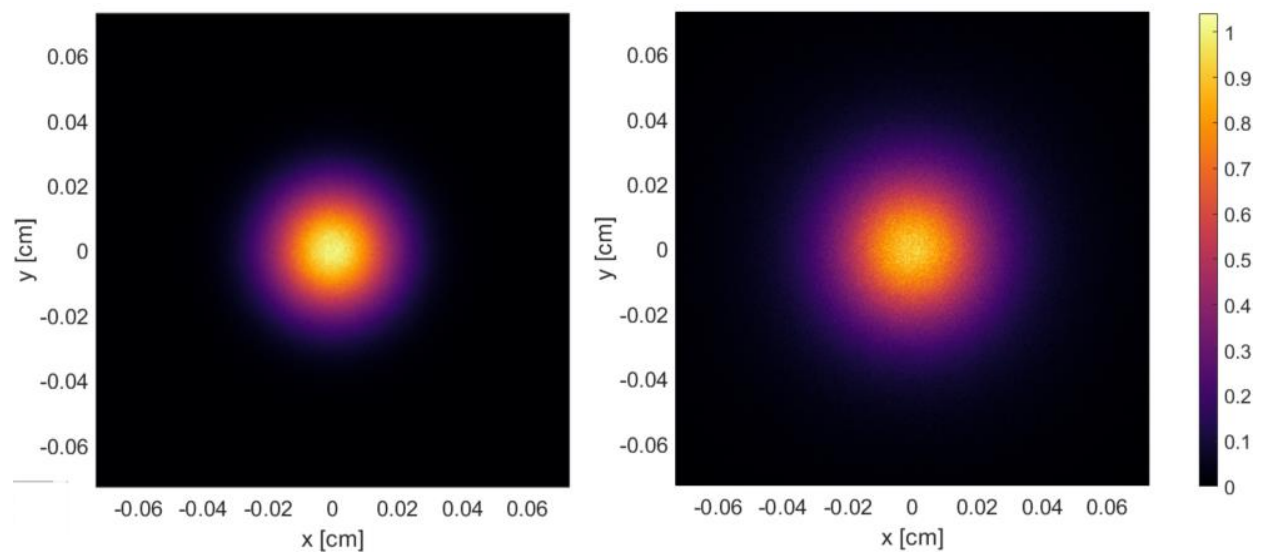

Figure 2. Normalized blue light (left) and luminescent light (right) fluence rates at the input surface of the phosphor. Note that the intensity scales are normalized.

Table 1. Material parameters used in the modeling of light distribution. The absorption coefficients are valid for blue light only.

\begin{tabular}{|c|l|l|l|l|l|}
\hline Phosphor & Thickness & $\left.\boldsymbol{\mu}_{\mathrm{a}} / \mathbf{c m}^{-\mathbf{1}} \mathbf{( 4 5 0} \mathbf{n m}\right)$ & $\boldsymbol{\mu}_{\mathbf{s}} / \mathbf{c m}^{-\mathbf{1}}$ & Front Surface & Rear surface \\
\hline 1 & $200 \mu \mathrm{m}$ & 100 & 0.001 & Polished & Air \\
\hline 2 & $250 \mu \mathrm{m}$ & 40 & 100 & Rough & Air \\
\hline 3 & $65 \mu \mathrm{m}$ & 300 & 100 & Rough & Air \\
\hline 4 & $250 \mu \mathrm{m}$ & 70 & 0.001 & Rough & Mirror \\
\hline
\end{tabular}

\section{EXPERIMENTAL SETUP}

The experimental setup for measuring the luminescent spot size is shown schematically in Figure 3. A laser diode at $450 \mathrm{~nm}$ (Nichia) was collimated and focused and the entire setup mounted on a translation stage. The beam size was measured through the focus using a beam scanner. The phosphor was positioned at the focus of the laser. By translating

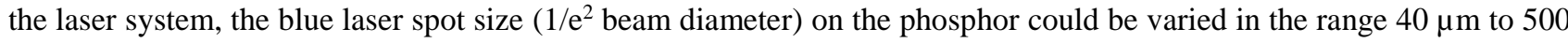
$\mu \mathrm{m}$, limited by access to measurements with the imaging system.

The imaging system used for measuring the luminescent spot size was constructed as a 4-f imaging system using 2 achromatic lenses with $100 \mathrm{~mm}$ focal length. The 4-f imaging system generated an image of the phosphor surface at the CMOS camera (Thorlabs DCC1545M, 5.2 $\mu \mathrm{m}$ pixel pitch). The imaging system was placed to image the input surface of the phosphor and with an angle of $30^{\circ}$ angle in order not to block the incident blue laser beam. Optical filters were placed in the imaging beam path to filter out residual blue light and attenuate the light in order not to saturate the camera. 


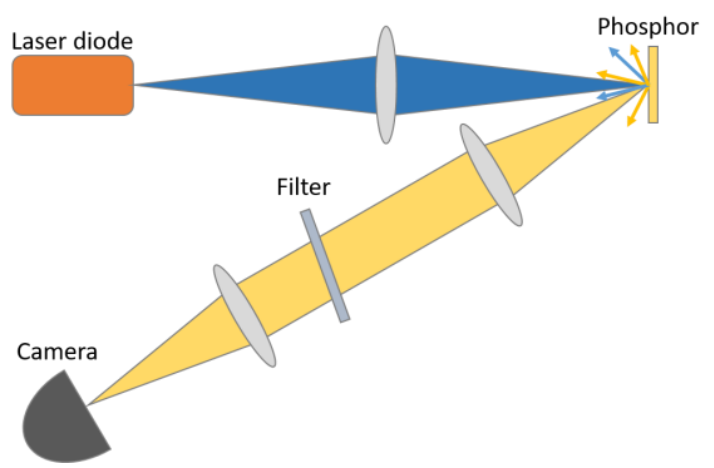

Figure 3. Schematic representation of the experimental setup.

A ceramic phosphor material was investigated experimentally. The phosphor was based on Ce:YAG as the luminescent material and had dimensions $10 \times 10 \times 0.25 \mathrm{~mm}^{3}$. The input surface of the phosphor was rough and scattering.

\section{RESULTS}

In the measurements, the luminescent spot size was measured using the imaging system and by varying the input blue laser spot size for the different phosphors, we investigated the influence of the blue laser spot size. In Figure 4 (a) and (b), the measured profiles of the luminescent spots are shown for two different input blue laser spot sizes. The luminescent spot is significantly wider than the incident blue laser spot. The center of the spot is only slightly larger than the blue laser spot but the shoulders of the spot are significantly enhanced making the effective spot size larger.

The simulated luminescent spot profiles are shown in Figure 4 (c) and (d) for phosphor 2 in Table 1 and they are in good agreement with the experimental results.

As seen from Figure 4, there is a large coupling between the luminescent spot size and the incident blue laser spot size. This was investigated by varying the input blue laser spot size and measuring the luminescent spot size. The experimental results for the ceramic phosphor material are shown in Figure 5 together with the simulated results for the different phosphors listed in Table 1. As expected, the luminescent spot size increases when the blue laser spot size is increased. The dependence between the blue and simulated luminescent spot sizes is seen to be different for the different phosphor material parameters. Phosphor 1, which is assumed transparent, have a luminescent spot size comparable to the blue spot, while the scattering phosphors 2-4 have a larger luminescent spot. The experimental results and the simulated results for phosphor 2 are in good agreement. 

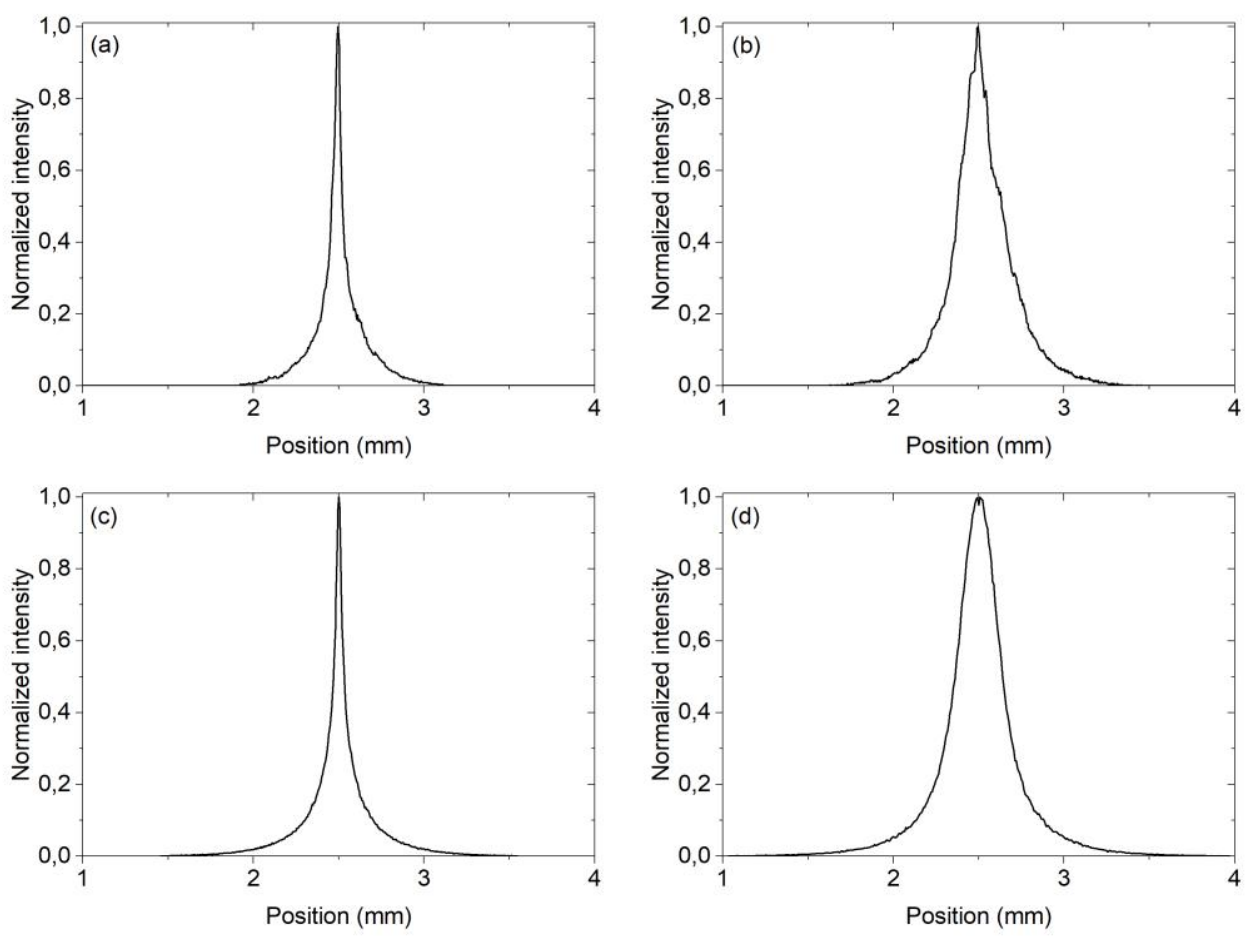

Figure 4. Luminescent spot profiles for two different input blue laser spot sizes of $40 \mu \mathrm{m}$ and $320 \mu \mathrm{m}$. (a) and (b) are measured spot profiles, while (c) and (d) are simulated profiles.

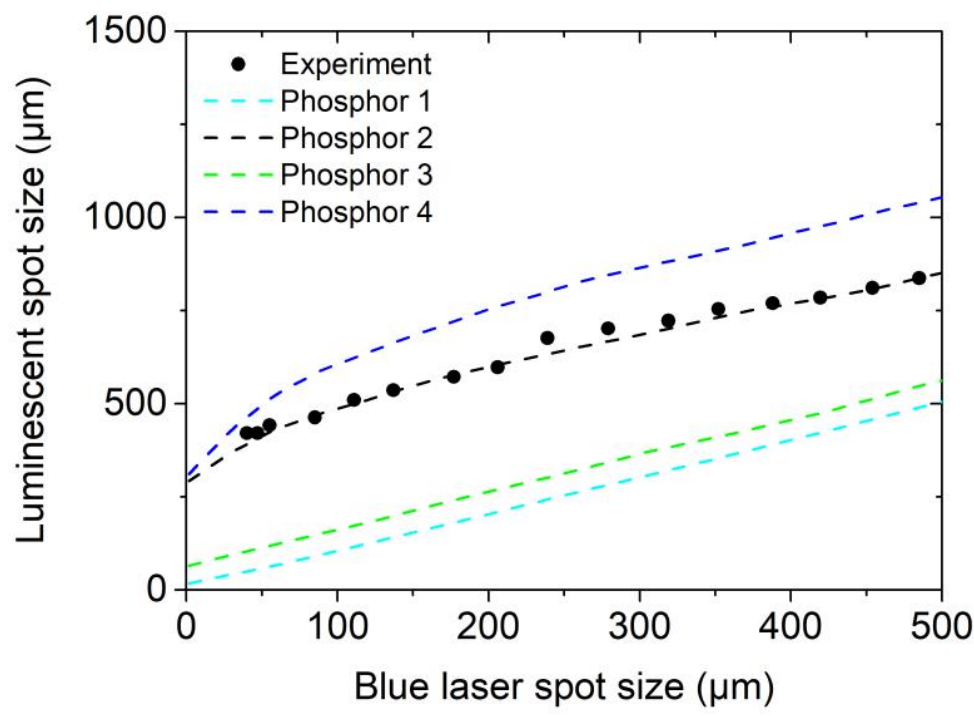

Figure 5. Measured (dots) and simulated (dashed lines) luminescent spot sizes vs. input blue laser spot size for different phosphor materials. The simulation results are for the phosphor parameters in Table 1.

The influence of the scattering and absorption coefficients on the luminescent spot size was investigated numerically and the results are shown in Figure 6. In the figure, a $200 \mu \mathrm{m}$ thick phosphor with a rough surface is assumed. From Figure 6 it is clear that the absorption coefficient has a large influence on the spot size, while the bulk scattering only has minor influence. 

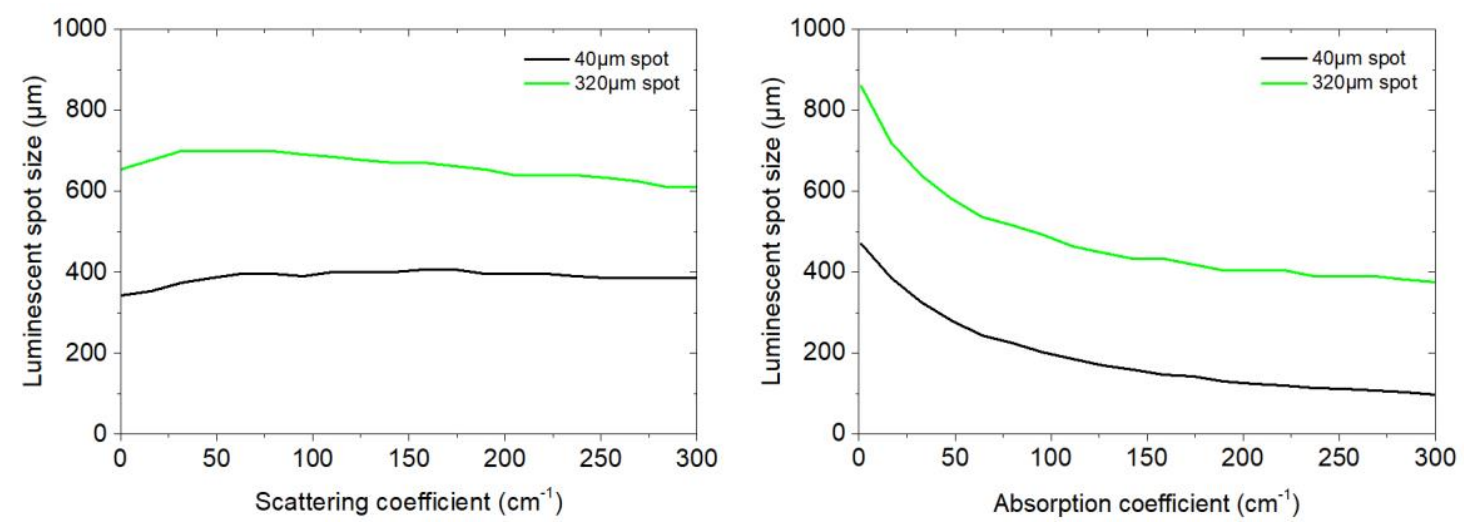

Figure 6. Dependence of the luminescent spot size on the scattering coefficient $\left(\mu_{\mathrm{a}}=30 \mathrm{~cm}^{-1}\right)$ (left) and absorption coefficient $\left(\mu_{\mathrm{s}}=100 \mathrm{~cm}^{-1}\right)$ (right) for two input blue laser spot sizes in a phosphor with rough surface.

\section{CONCLUSION}

The simulation results show significant differences in the achieved luminescent spot size for a given input blue laser spot for different phosphor material parameters. It is evident that a transparent phosphor will provide the smallest luminescent spot size, while scattering and surface texturing enlarges the luminescent spot. A significant disadvantage of transparent phosphors is the poor color mixing, as the un-absorbed blue light is transmitted straight through. Therefore, the scattering phosphors are more interesting from an application point of view. However, the luminescent spot size emitted from these phosphors is highly dependent on the material properties and will be larger than the incident laser spot.

The larger luminescent spot size in phosphor materials limits the luminous exitance from a phosphor. A larger spot size complicates light collection and collimation and will result in a larger divergence of the light cone or reduced transmission through the optical system. For specific application requirements, it may be necessary to design the phosphor material to the desired properties. The findings of this work can assist in selecting and developing phosphor materials for laser lighting for highest luminance lighting.

\section{ACKNOWLEDGEMENT}

This research was supported by DTU Fotonik and the Danish Energy Technology Development and Demonstration program (EUDP) (64017-0588).

\section{REFERENCES}

1. J. J. Wierer, J. Y. Tsao, and D. S. Sizov, "Comparison between blue lasers and light-emitting diodes for future solid-state lighting," Laser Photon. Rev. 7, 963-993 (2013).

2. V. J. Nadeau, D. S. Elson, G. B. Hanna, and M. A. A. Neil, "Modelling of a laser-pumped light source for endoscopic surgery," in Proc. of SPIE (2008), Vol. 7103, p. 71030J.

3. L. Ulrich, "Audi Pixelated Laser Headlights Light the Road and Paint It Too," http://spectrum.ieee.org/cars-thatthink/transportation/advanced-cars/audi-lights-the-road-with-pixelated-laser-headlights.

4. U. Hartwig and M. Bruemmer, "Challenges for reducing the size of laser activated remote phosphor light engines for DLP projection," Int. Opt. Des. Conf. 2014 9293, 929313 (2014).

5. T. W. Kang, K. W. Park, J. H. Ryu, S. G. Lim, Y. M. Yu, and J. S. Kim, "Strong thermal stability of Lu3A15O12:Ce3+single crystal phosphor for laser lighting," J. Lumin. 191, 35-39 (2017).

6. J. Xu, A. Thorseth, C. Xu, A. Krasnoshchoka, M. Rosendal, C. Dam-Hansen, B. Du, Y. Gong, and O. B. Jensen, "Investigation of laser-induced luminescence saturation in a single-crystal YAG:Ce phosphor: Towards unique architecture, high saturation threshold, and high-brightness laser-driven white lighting," J. Lumin. 212, 279-285 (2019). 
7. J. Xu, J. Wang, Y. Gong, X. Ruan, Z. Liu, B. Hu, B. Liu, H. Li, X. Wang, and B. Du, "Investigation of an LuAG:Ce translucent ceramic synthesized via spark plasma sintering: Towards a facile synthetic route, robust thermal performance, and high-power solid state laser lighting," J. Eur. Ceram. Soc. 38, 343-347 (2018).

8. M. Xu, J. Chang, J. Wang, C. Wu, and F. Hu, "Al2O3-YAG:Ce composite ceramics for high-brightness lighting," Opt. Express 27, 872-885 (2019).

9. P. Zheng, S. Li, L. Wang, T. L. Zhou, S. You, T. Takeda, N. Hirosaki, and R. J. Xie, "Unique Color Converter Architecture Enabling Phosphor-in-Glass (PiG) Films Suitable for High-Power and High-Luminance LaserDriven White Lighting," ACS Appl. Mater. Interfaces 10, 14930-14940 (2018).

10. E. F. Schubert, Light-Emitting Diodes, Second Edi (Cambridge University Press, 2006).

11. J. Ueda, P. Dorenbos, A. J. J. Bos, A. Meijerink, and S. Tanabe, "Insight into the Thermal Quenching Mechanism for Y3A15O12:Ce3+ through Thermoluminescence Excitation Spectroscopy," J. Phys. Chem. C 119, 25003-25008 (2015).

12. S. You, S. Li, P. Zheng, T. Zhou, L. Wang, L. Liu, N. Horisaki, F. Xu, and R.-J. Xie, "A Thermally Robust La3Si6N11:Ce-in-Glass Film for High-Brightness Blue-Laser-Driven Solid State Lighting," Laser Photon. Rev. 13, 1800216 (2019).

13. A. Lenef, J. Kelso, Y. Zheng, and M. Tchoul, "Radiance limits of ceramic phosphors under high excitation fluxes," Curr. Dev. Lens Des. Opt. Eng. XIV 8841, 884107 (2013).

14. A. Krasnoshchoka, A. Thorseth, C. Dam-Hansen, D. D. Corell, P. M. Petersen, and O. B. Jensen, "Investigation of saturation effects in ceramic phosphors for laser lighting," Materials (Basel). 10, 1407 (2017).

15. Q.-Q. Zhu, X.-J. Wang, L. Wang, N. Hirosaki, T. Nishimura, Z.-F. Tian, Q. Li, Y.-Z. Xu, X. Xu, and R.-J. Xie, " $\beta$-Sialon: Eu phosphor-in-glass: a robust green color converter for high power blue laser lighting," J. Mater. Chem. C 3, 10761-10766 (2015).

16. S. Li, L. Wang, N. Hirosaki, and R. J. Xie, "Color Conversion Materials for High-Brightness Laser-Driven Solid-State Lighting," Laser Photonics Rev. 1800173, 1-29 (2018).

17. A. Lenef, J. Kelso, M. Tchoul, O. Mehl, J. Sorg, and Y. Zheng, "Laser-activated remote phosphor conversion with ceramic phosphors," 9190, 91900C (2014).

18. A. Correia, P. Hanselaer, and Y. Meuret, "An Efficient Optothermal Simulation Framework for Optimization of High-Luminance White Light Sources," IEEE Photonics J. 8, 1601215 (2016).

19. Y. Ma, W. Lan, B. Xie, R. Hu, and X. Luo, "An optical-thermal model for laser-excited remote phosphor with thermal quenching," Int. J. Heat Mass Transf. 116, 694-702 (2018).

20. P. Zheng, S. Li, R. Wei, L. Wang, T. Zhou, Y. Xu, T. Takeda, N. Hirosaki, and R. Xie, "Unique Design Strategy for Laser-Driven Color Converters Enabling Superhigh-Luminance and High-Directionality White Light," Laser Photon. Rev. 13, 1900147 (2019).

21. D. Marti, R. N. Aasbjerg, P. E. Andersen, and A. K. Hansen, "MCmatlab: an open-source, user-friendly, MATLAB-integrated three-dimensional Monte Carlo light transport solver with heat diffusion and tissue damage," J. Biomed. Opt. 23, 121622 (2018).

22. M. L. Meretska, R. Uppu, G. Vissenberg, A. Lagendijk, W. L. Ijzerman, and W. L. Vos, "Analytical modeling of light transport in scattering materials with strong absorption," Opt. Express 25, A906 (2017).

23. S. Nishiura, S. Tanabe, K. Fujioka, and Y. Fujimoto, "Properties of transparent Ce:YAG ceramic phosphors for white LED," Opt. Mater. (Amst). 33, 688-691 (2011). 